\title{
Acousto-Resonance Spectroscopy of Nonlinear-Optical Crystals in Process of Laser Frequency Conversion
}

\author{
O. A. Ryabushkin ${ }^{1,2}$, A. V. Konyashkin ${ }^{1,2}$, D. V. Myasnikov ${ }^{1,2}$, V. A. Tyrtyshnyy ${ }^{2}$, \\ O. I. Vershinin ${ }^{1,2}$, A. I. Baranov ${ }^{1,2}$ \\ ${ }^{1}$ Moscow Institute of Physics and Technology, Institutskiy per. 9, Dolgoprudnyy, Russia \\ ${ }^{2}$ NTO “IRE-Polus", Vvedensky Sq.1, Fryazino, Russia \\ Email: roa228@mail.ru
}

Received 2013

\begin{abstract}
Dependence of the periodically poled nonlinear-optical lithium niobate (PPLN) crystal temperature on laser power in the course of laser frequency conversion was measured using piezoelectric resonance. Crystal's temperature tuning curves are precisely measured using concept of the equivalent temperature. Both optical absorption and heat transfer coefficients of the crystal are measured employing kinetics of the crystal equivalent temperature.
\end{abstract}

Keywords: Piezoelectric Resonance; Nonlinear-Optical Crystals; Second Harmonic Generation; Equivalent Temperature

\section{Introduction}

Nonlinear-optical crystals are widely used in various nonlinear-optical processes [1-6]. Due to optical absorption any interaction of the laser radiation with crystals results in its heating. In high-power laser applications optical absorption can cause crystal structural defects formation and even its destruction $[7,8]$.

It is well known that efficient nonlinear frequency conversion occurs when phase matching condition is fulfilled for wavelengths involved [1]. Here the crystal temperature control plays crucial role because temperature deviation from the optimum point results in efficiency decreasing. Pump power increase leads to crystal overheating and phase matching violation. In Second Harmonic Generation (SHG) experiments optimum temperature is usually determined by adjusting the thermostat temperature while monitoring the output second harmonic power.

It is well known that most crystals applied in nonlinear optics possess piezoelectric properties. External radiofrequency electric field excites acoustical vibrations in such crystals due to indirect piezoelectric effect. When external electric field frequency coincides with one of the crystal internal acoustical mode frequency the piezoelectric resonance is excited.

Strongly temperature-sensitive piezoelectric resonance frequency is a powerful tool for research of nonlinearoptical crystals interaction with laser radiation. It was demonstrated that such important properties as ionic conductivity $[9,10]$ and optical absorption [11-13] can be precisely measured using crystal piezoelectric resonances.

Novel method based on the piezoelectric resonance spectroscopy technique was proposed for the accurate temperature determination of nonlinear-optical crystal interacting with laser radiation $[12,13]$.

It was demonstrated that for the extremely wide range of laser power values the internal crystal temperature can be directly determined by measuring its piezoelectric resonance frequency shift $[12,13]$. Thus measured temperature is called crystal equivalent temperature $\theta_{\mathrm{eq}}$. In this paper we present results of the periodically poled nonlinear-optical lithium niobate (PPLN) crystals temperature measurement in the course of the second harmonic generation of the $\mathrm{CW}$ single-mode ytterbium-doped fiber laser radiation.

\section{Experiment}

\subsection{Crystal Samples}

Two specimens of PPLN crystals were used in the experiment. Both are rectangular parallelepipeds with dimensions $1 \times 2.6 \times 4.9 \mathrm{~mm}^{3}$ (PPLN1) and $1 \times 3 \times 10 \mathrm{~mm}^{3}$ (PPLN2) respectively. Crystals are poled along z-axis (1 $\mathrm{mm}$ ) and quasi-phase-matched for SHG of the $1064 \mathrm{~nm}$ wavelength. Crystal's input and output facets are anti-reflective coated for both $532 \mathrm{~nm}$ and $1064 \mathrm{~nm}$.

\subsection{Experimental Setup}

Simplified block-scheme of the experimental setup for the nonlinear-optical crystal's electrical impedance measure- 
ments during interaction with laser radiation is shown in Figure 1. Crystal sample is placed in unclamped manner onto silica fibers between two strip metallic electrodes that form the capacitor. Voltage of the frequency $f$ from the radiofrequency (RF) generator is applied to the capacitor that is connected in series with the load resistor $\mathrm{R}$ $=50$ Ohm. Capacitor with crystal in it is placed inside the thermostat. Temperature stabilization is better than $50 \mathrm{mK}$. Crystal response to the applied electric field is analyzed by measuring spectra of the voltage amplitude $\left|U_{R}\right|$ and phase $\varphi$ on the load resistor via lock-in amplifier. These signals are proportional to the amplitude and phase of the electrical current flowing through the crystal. Thus crystal electrical admittance can be calculated. At the certain frequency $R f$ piezoelectric resonance between the external electric field and one of the crystal internal vibration modes can be observed.

$\mathrm{CW}$ laser source is polarized single-mode ytterbiumdoped fiber laser. Operating wavelength is $\lambda_{1}=1064 \mathrm{~nm}$, spectral line width is $0.1 \mathrm{~nm}$, output power is up to $25 \mathrm{~W}$. Beam quality factor $\mathrm{M}^{2}$ is 1.05 . Laser polarization degree is $20 \mathrm{~dB}$. Laser beam is focused inside the crystal. Beam waist is $30 \mu \mathrm{m}$. Beam splitter is used for separating pump $\lambda_{1}=532 \mathrm{~nm}$ and orthogonally polarized second harmonic $\lambda_{2}=532 \mathrm{~nm}$ radiation.

Temperature calibration of the piezoelectric resonance frequencies should be performed prior to the investigation of the crystal interaction with laser radiation. Figure 2 shows dependencies of the $U_{R}$ voltage phase $\varphi$ on frequency $f$ measured near PPLN1 piezoelectric resonance for two temperatures. Piezoelectric resonance frequency $R f_{i}$ at each temperature corresponds to the phase minimum.

\section{Crystal Equivalent Temperature}

When crystal is uniformly heated its resonance frequency $R f_{i}$ linearly depends on crystal temperature $\theta_{c r}$ with the piezoelectric thermal coefficient $K_{i}^{\text {prt }}$ of the particular $i$-th vibration mode $[12,13]$ :

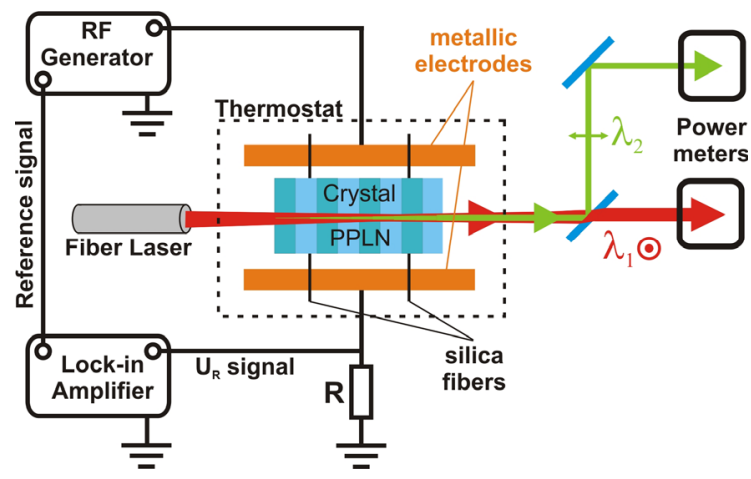

Figure 1. Block scheme of the experimental setup for measuring piezoelectric resonances of the nonlinear-optical crystal during its interaction with laser radiation.

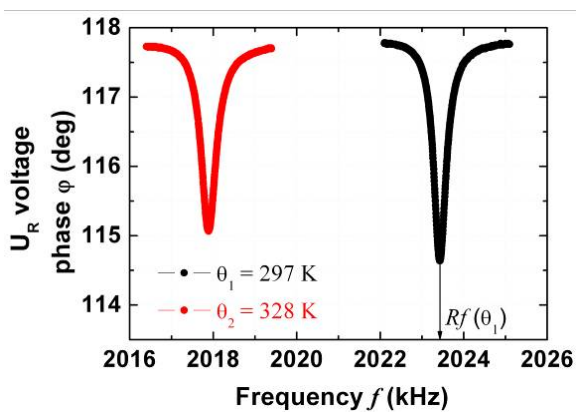

Figure 2. Frequency dependencies of the $U_{R}$ voltage phase measured near PPLN1 piezoelectric resonances for two crystal temperatures.

$$
R f_{i}\left(\theta_{c r}\right)=R f_{i}\left(\theta_{0}\right)+K_{i}^{p r t}\left(\theta_{c r}-\theta_{0}\right) .
$$

here $\theta_{0}$ is initial crystal temperature.

When crystal is nonuniformly heated by laser radiation its temperature distribution can be obtained by solving heat conduction equation using appropriate boundary condition at the crystal-air interface. In assumption of weak optical absorption and crystal convective air cooling it can be written as follows

$$
\left\{\begin{array}{l}
\kappa_{c r}\left(\frac{\partial^{2}}{\partial x^{2}}+\frac{\partial^{2}}{\partial y^{2}}\right) \theta+\alpha(\lambda) I(x, y)=0, \\
-\kappa_{a} \frac{\partial \theta}{\partial n}=h^{T}\left(\theta_{\text {in }}-\theta_{\text {out }}\right) .
\end{array},\right.
$$

here $\kappa_{c r}$ and $\kappa_{a}$ are thermal conductivities of the crystal and air respectively; $I(x, y)$ is radiation intensity distribution; $\alpha(\lambda)$ is optical absorption coefficient, which is wavelength dependent; $\theta_{\text {in }}$ and $\theta_{\text {out }}$ are temperatures at the interface of crystal and air respectively; $n$ is the normal vector to the interface; $h^{T}$ is the heat transfer coefficient.

It was demonstrated earlier that piezoelectric resonance frequencies dependence on laser power $P_{p}$ can be characterized by piezoelectric resonance optical coefficients $K_{i}^{\text {pro }}[12,13]$.

Then temperature of the laser heated crystal can be precisely determined using notion of crystal equivalent temperature. Crystal equivalent temperature is expressed as follows

$$
\theta_{\mathrm{eq}}\left(P_{p}\right)=\theta_{0}+\Delta \theta_{\mathrm{eq}}\left(P_{p}\right)
$$

Here $\theta_{0}$ is crystal temperature at $P_{p}=0$ and $\Delta \theta_{\mathrm{eq}}\left(P_{p}\right)$ is crystal equivalent heating temperature determined directly from the $i$-th piezoelectric resonance frequency shift

$$
\Delta \theta_{\text {eq }}\left(P_{p}\right)=\frac{R f_{i}\left(P_{p}\right)-R f_{i}(0)}{K_{i}^{p r t}} .
$$

For the linear case of $R f_{i}$ dependence on power $P_{p}$ it equals

$$
\Delta \theta_{\mathrm{eq}}\left(P_{p}\right)=\frac{K_{i}^{\text {pro }}}{K_{i}^{\text {prt }}} P_{p}=\beta P_{p} .
$$


Here $\beta$ is piezoelectric resonance optothermal coefficient. Value of $\beta$ depends on crystal optical absorption $\alpha(\lambda)$, polarization of laser radiation and heat exchange conditions. However, in case of nonlinear-optical frequency conversion crystal is heated nonlinearly. Still its temperature can be determined using relations (3) and (4).

\section{Experimental Results}

\subsection{Stationary Measurements of Crystal Equivalent Temperature}

Piezoelectric resonance thermal coefficients were measured using eq. (1) for two resonances observed in PPLN1 and PPLN2 crystals. Parameters of the piezoelectric resonances selected for detailed investigation are listed in Table 1.

Crystal equivalent temperature $\theta_{\mathrm{eq}}$ was measured for each pump laser power $P_{p}$ value after reaching stationary temperature state of the crystal with surrounding air. Thermostat temperature was fixed at $20^{\circ} \mathrm{C}$. It was observed that when laser polarization is perpendicular to the crystal poling axis no second harmonic is generated and crystal equivalent temperature linearly depends on pump power for both crystals. In case laser polarization $e_{p}$ is parallel to the crystal poling axis second harmonic can be efficiently generated when crystal is at phase matching temperature. Results of crystal's equivalent temperature measurement for the case of pump power subsequent increase from 0 to its maximum are presented in Figure 3.

Table 1. Crystal piezoelectric resonances.

\begin{tabular}{ccc}
\hline \multirow{2}{*}{ Crystal } & \multicolumn{2}{c}{ Piezoelectric resonance parameters } \\
\cline { 2 - 3 } & $R f$ at $\theta_{0}=297 \mathrm{~K}, \mathrm{kHz}$ & $K^{p r t}, \mathrm{~Hz} / \mathrm{K}$ \\
\hline PPLN1 & 2023.4 & -179 \\
PPLN2 & 2006.6 & -150 \\
\hline
\end{tabular}

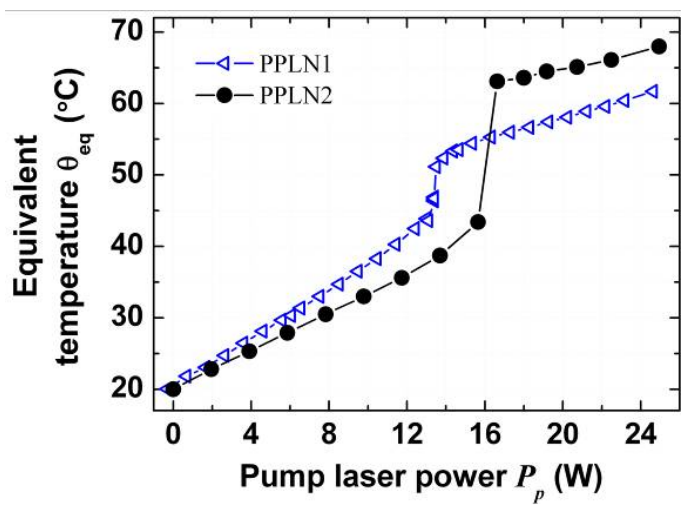

Figure 3. Dependencies of the equivalent temperature on laser power measured for PPLN1 and PPLN2 crystals.
As it can be seen for low $P_{p}$ values both PPLN1 and PPLN2 are linearly heated with coefficients $\beta_{1}=1.7 \mathrm{~K} / \mathrm{W}$ and $\beta_{2}=1.3 \mathrm{~K} / \mathrm{W}$ respectively. Above certain power value $\left(P_{p}=13.4 \mathrm{~W}\right.$ for PPLN1, $P_{p}=15.6 \mathrm{~W}$ for PPLN2) abrupt temperature rise occurs because crystal temperature approaches to the corresponding phase matching temperature. These points are unstable because small increase of $P_{p}$ leads to rapid rise of both second harmonic power $P_{s h}$ and crystal temperature (see Figure 4). PPLN1 crystal stationary temperature point at $P_{p}=13.5 \mathrm{~W}$ is slightly less than its phase matching temperature $\theta_{\mathrm{pm}}$. PPLN2 has higher value of $\theta_{\mathrm{pm}}$. So that here break point is observed at higher $P_{p}$ value. Second harmonic power rose from $P_{s h}=50 \mathrm{~mW}$ at $P_{p}=15.6 \mathrm{~W}$ reaching $750 \mathrm{~mW}$ and resulted in stationary $P_{s h}=150 \mathrm{~mW}$ at $P_{p}=16.6 \mathrm{~W}$. It means that PPLN2 temperature overcame the phase matching temperature. Further power increase results in moderate $\theta_{\text {eq }}$ rise. If now after reaching its maximum pump power is subsequently decreased then the temperature break point occurs at lower $P_{p}$ value.

Experimental results of $\theta_{\mathrm{eq}}$ and $P_{s h}$ dependence on $P_{p}$ measured for PPLN2 when $P_{p}$ is lowered are presented in Figures 5 and 6. Here crystal temperature is once again approaches to the phase matching temperature at $P_{p}=12$ $\mathrm{W}$ (Figure 5). At the next point $P_{p}=11.4 \mathrm{~W}$ PPLN2

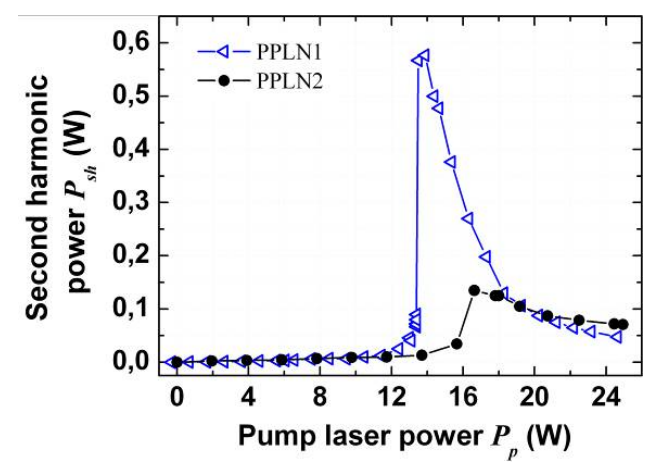

Figure 4. Dependencies of the second harmonic power on the pump power measured for PPLN1 and PPLN2.

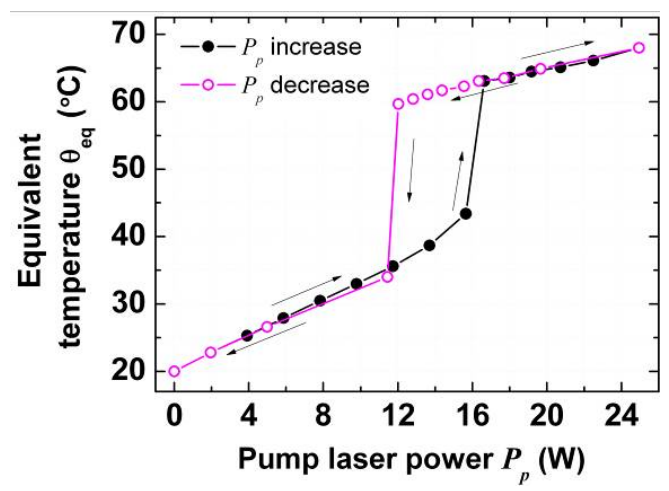

Figure 5. Dependencies of PPLN2 equivalent temperature on pump power measured for $\boldsymbol{P}_{p}$ increase to maximum and subsequent decrease to zero. 
crystal temperature is considerably lower than phase matching temperature. Second harmonic power nonlinearly rose from $P_{s h}=70 \mathrm{~mW}$ at $P_{p}=25 \mathrm{~W}$ to $P_{s h}=350$ $\mathrm{mW}$ at $P_{p}=12 \mathrm{~W}$ and then fell to $P_{s h}=10 \mathrm{~mW}$ at $P_{p}=$ 11.4 W (see Figure 6).

True temperature tuning curves of the PPLN crystals can be also precisely measured using concept of equivalent temperature. In this case thermostat temperature should be tuned when pump power is fixed. Results obtained for PPLN1 crystal are presented in Figure 7. PPLN1 phase matching temperature decreases with power: $\mathrm{d} \theta_{\mathrm{pm}} / \mathrm{d} P_{p}=-0.11 \mathrm{~K} / \mathrm{W}$. Crystal temperature acceptance bandwidth is almost the same for these $P_{p}$ values: $\Delta \theta_{\mathrm{pm}}=$ $6^{\circ} \mathrm{C}$.

\subsection{Crystal Equivalent Temperature Kinetics Measurements}

Conventional method for the precise determination of optical absorption coefficients of nonlinear-optical crystals is laser calorimetry [14]. It is based on measurements of the heating kinetics of air near the crystal surface during and after laser irradiation. Both optical absorption $\alpha$

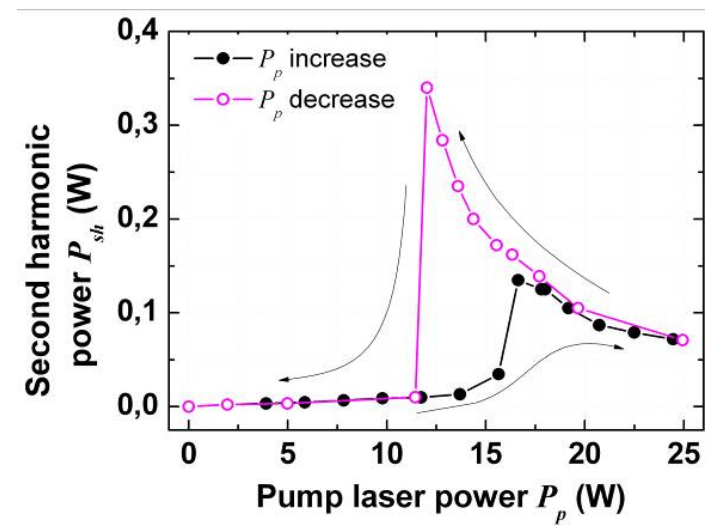

Figure 6. Dependencies for $e_{p} \| Z_{c r}$ of the second harmonic power on the pump laser power measured for $P_{p}$ increase to maximum and subsequent decrease to zero.

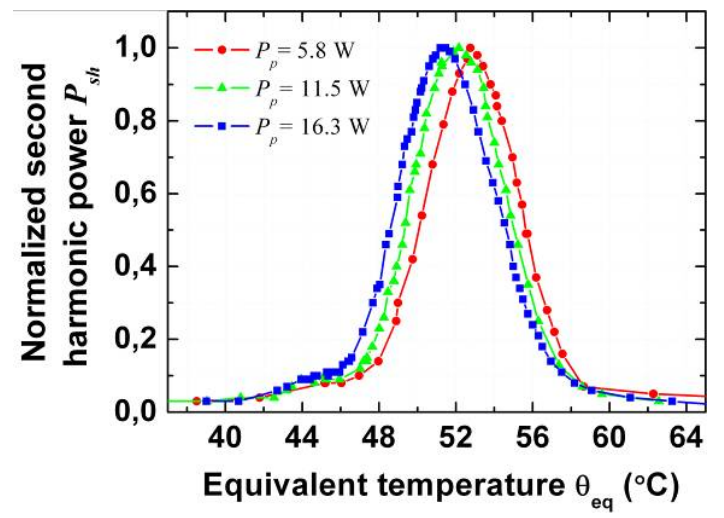

Figure 7. Temperature tuning curves measured for PPLN1 at different pump powers. and heat transfer $h^{\mathrm{T}}$ coefficients are then calculated by solving nonstationary heat conduction equation taking into account boundary conditions. To the present day there are no simple direct methods for the precise crystal temperature measurement during interaction with laser radiation.

Novel modification of calorimetry technique that we propose is to use concept of the crystal equivalent temperature for measuring temperature kinetics of the laser heated crystal. Crystal equivalent temperature kinetics is directly measured using resonance frequency shift $\Delta R f$ dependence on time $t$ when the laser power is switched on. Here we use the same experimental setup. As it is shown in Figure 8 the RF generator frequency $f$ is changed stepwise (step $\Delta f$ ) and phase response $(\varphi)$ minimum that corresponds to $R f$ at moment $\mathrm{t}_{\mathrm{i}}$ is measured in each $\Delta \mathrm{t}$ interval. Characteristic $R f$ kinetics time constant $\tau$ is obtained using fitting function

$$
R f(t)=\left[R f(t)-R f\left(P_{p}\right)\right] \exp (-t / \tau)+R f\left(P_{p}\right) .
$$

Here $R f\left(P_{p}\right)$ corresponds to the temperature stationary point for the given power $P_{p}$. Obviously crystal equivalent temperature kinetics $\tau$ value is identical to that of $R f$ kinetics. Then heat transfer coefficient is obtained:

$$
h^{T} S=\frac{m c}{\tau} \text {. }
$$

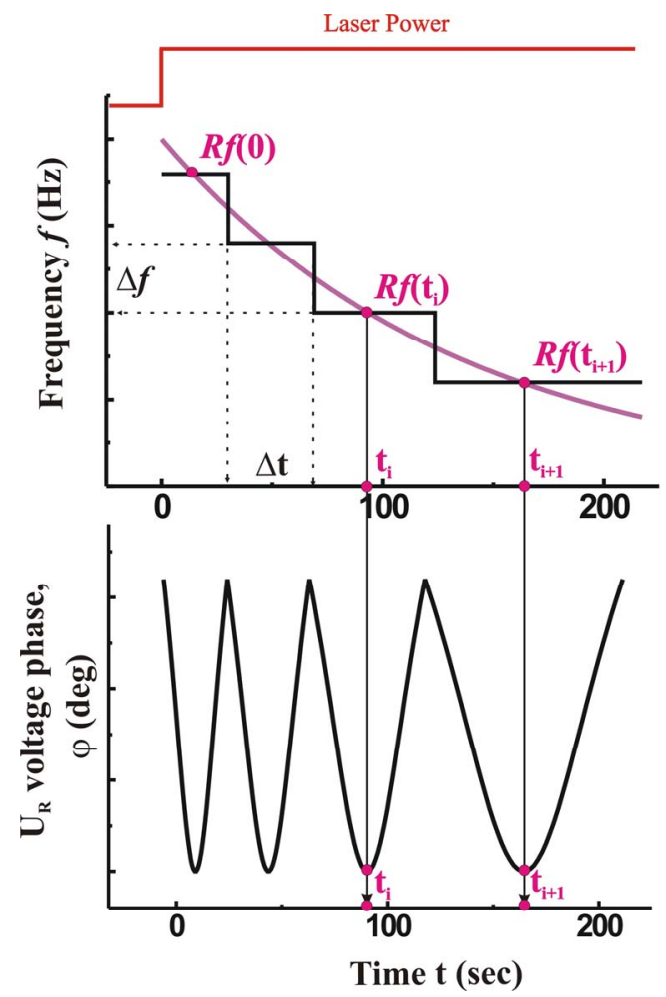

Figure 8. Experiment of $R f$ kinetics measurement: time dependence of $\boldsymbol{R} \boldsymbol{f}$ (corresponds to $\varphi$ minimum) is measured via stepwise change of the generator frequency $f$. 
here $m$ is crystal mass, $c$ is specific heat capacity, $S$ is crystal surface area. Optical absorption coefficient is calculated as follows

$$
\alpha(\lambda) L=h^{T} S \beta
$$

here $L$ is crystal length. Equivalent tempearature kinetics measured for PPLN1 crystal for $P_{p}=5.6 \mathrm{~W}$ is shown in Figure 9. Values of $h^{T}$ and $\alpha(\lambda) L$ obtained for PPLN1 crystal $(L=4.9 \mathrm{~mm})$ are summarized in Table 2 .

\section{Discussion and Conclusions}

Novel powerful method of acousto-resonant spectroscopy employing concept of crystal equivalent temperature was applied for precise PPLN temperature measurement during nonlinear-optical interaction with laser radiation.

Hysteresis of PPLN temperature and optical bistability of second harmonic power in respect to pump power were observed in the course of second harmonic generation.

Temperature tuning curves of nonlinear-optical crystals can be precisely measured using concept of the equivalent temperature. Rate of phase matching temperature decrease with pump power measured for PPLN crystal $\mathrm{d} \theta_{\mathrm{pm}} / \mathrm{d} P_{p}=-0.11 \mathrm{~K} / \mathrm{W}$ is of the same order as that obtained for MgO:sPPLT crystal $\left(\mathrm{d} \theta_{\mathrm{pm}} / \mathrm{d} P_{p}=-0.14 \mathrm{~K} / \mathrm{W}\right)$ [4].

Crystal equivalent temperature kinetics during its interaction with laser radiation is employed for the accurate measurement of both crystal optical absorption and heat transfer coefficients.

Our experiments reveal that temperature control of crystals interacting with laser radiation via acoustic resonance

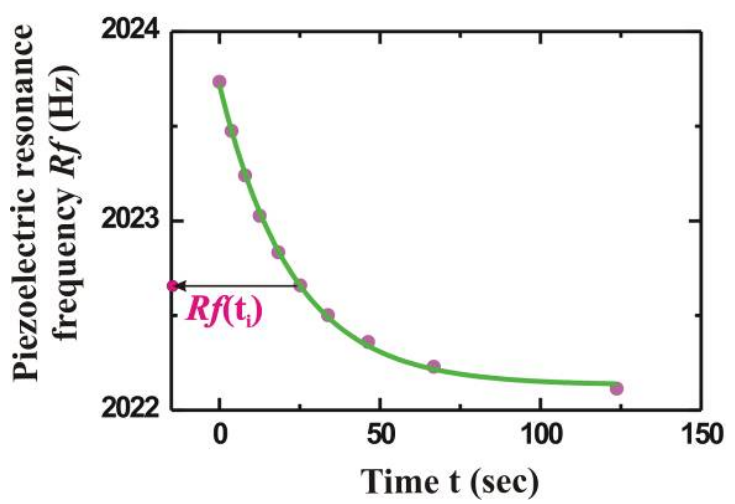

Figure 9. Kinetics of piezoelectric resonance frequency $R f$ measured for laser heating of PPLN1 crystal.

Table 2. Results of laser heated PPLN temperature kinetics.

\begin{tabular}{cccc}
\hline \multirow{3}{*}{ Crystal } & \multicolumn{3}{c}{ Table Column Head } \\
\cline { 2 - 4 } & $\tau, \mathrm{s}$ & $h^{T}, \mathrm{~W} / \mathrm{m}^{2} \mathrm{~K}$ & $\alpha(\lambda) L$ \\
\hline PPLN1 & 22.2 & 43 & $2.9 \times 10^{-3}$ \\
\hline
\end{tabular}

techniques is restricted only by laser powers that lead to crystal optical damage.

\section{REFERENCES}

[1] A. V. G. Dmitriev and L. V. Tarasov, "Applied Nonlinear Optics," Fizmatlit, Moscow (in Russian), 2004.

[2] F. Kienle, D. Lin, S.-ul. Alam, et al., "Green-pumped, Picosecond MgO: PPLN Optical Parametric Oscillator," Journal of the Optical Society of America B, Vol. 29, No. 1, 2012, pp. 144-152. doi:10.1364/JOSAB.29.000144

[3] H. Furuya, A. Morikawa, K. Mizuuchi and K. Yamamoto, "High Beam-Quality Continuous Wave 3W Green- Light Generation in Bulk Periodically Poled $\mathrm{MgO}$ : $\mathrm{LiNbO}_{3}$," Japanese Journal of Applied Physics Part 1, Vol. 45, 2006, pp. 6704-6707. doi:10.1143/JJAP.45.6704

[4] S. C. Kumar, G. K. Samanta and M. Ebrahim-Zadeh, "High-Power, Single-Frequency, Continuous-Wave Second-Harmonic-Generation of Ytterbium Fiber Laser in PPKTP and MgO: sPPLT," Optics Express, Vol. 17, No. 16, 2009, pp. 13711-13726. doi:10.1364/OE.17.013711

[5] S. C. Kumar, G. K. Samanta, K. Devi and M. EbrahimZadeh, "High-Efficiency, Multicrystal, Single-Pass, Continuous-Wave Second Harmonic Generation," Optics Express, Vol. 19, No. 12, 2011, pp. 1152-1169. doi:10.1364/OE.19.011152

[6] V. P. Gapontsev, V. A. Tyrtyshnyy, O. I. Vershinin, B. L. Davydov and D. A. Oulianov, "Third Harmonic Frequency Generation by Type-I Critically Phase-Matched $\mathrm{LiB}_{3} \mathrm{O}_{5}$ Crystal By Means of Optically Active Quartz Crystal," Optics Express, Vol. 21, No. 3, 2013, pp. 3715-3720. doi:10.1364/OE.21.003715

[7] H. Yoshida, H. Fujita, M. Nakatsuka, et al., "Dependences of Laser-Induced Bulk Damage Threshold and Crack Patterns in Several Nonlinear Crystals on Irradiation Direction," Japanese Journal of Applied Physics, Vol. 45, No. 2a, 2006, pp. 766-769. doi:10.1143/JJAP.45.766

[8] A. Hildenbrand, F. R. Wagner, H. Akhouayri, et al, "Laser-Induced Damage Investigation at $1064 \mathrm{~nm}$ in $\mathrm{KTiOPO}_{4}$ crystals and its analogy with $\mathrm{RbTiOPO}_{4}$," Applied Optics, Vol. 48, No. 21, 2009, pp. 4263-4269. doi:10.1364/AO.48.004263

[9] A. V. Konyashkin, V. A. Doronkin, V. A. Tyrtyshnyy, et al., "Resonant Acoustic Calorimetry of the Interaction of Laser Radiaiton with Nonlinear-Optical Crystals," 2009 IEEE International Ultrasonic Symposium Proceedings, 2009, pp. 2045-2048. doi:10.1109/ULTSYM.2009.5441994

[10] R. A. Theerthan, B. Menaert, B. Boulanger and M. Maglione, "Linking Ionic Conductivity and Piezoelectric Resonance in $\mathrm{KTiOPO}_{4}$," Physical Review B, Vol. 85, 2012, pp. 024103(1-8).

[11] F. Bezancon, J. Mangin, P. Stimer, M. Maglione, “Accurate Determination of Weak Optical Absorption of Piezoelectric Crystals Used as Capacitive Massive Bolometers," IEEE Journal of Quantum Electronics, Vol. 37, No. 11, 2001, pp. 1396-1400. doi:10.1109/3.958353

[12] O. A. Ryabushkin, D.V. Myasnikov, A. V. Konyashkin 
and V. A. Tyrtyshnyy, "Equivalent Temperature of Nonlinearoptical Crystals Interacting with Laser Radiation," Journal of the European Optical Society-Rapid publications, Vol. 6, 2011, pp. 11032(1-8).

[13] O. A. Ryabushkin and D. V. Myasnikov, "Experimental Determination and The Theoretical Model of An Equivalent Temperature of Nonlinear Optical Crystals Interact- ing With High-Power Laser Radiation," Quantum Electron, Vol. 42, No. 6, 2012, pp. 539-544. doi:10.1070/QE2012v042n06ABEH014657

[14] ISO 11551: test method for absorptance of optical laser components / International Organization for Standartization. Geneva Switzerland, 2003. 